\title{
Cytoplasmic male sterility in Drosophila melanogaster associated with a mitochondrial CYTB variant
}

Heredity (2011) 107, 374-376; doi:10.1038/hdy.2011.12; published online 16 March 2011

Somewhat buried in a paper investigating mitochondrial genetic variation and ageing in Drosophila melanogaster (Clancy, 2008) was a serendipitous finding; cytoplasmic male sterility involving naturally occurring mitonuclear incompatibility, associated with a single polymorphism in mitochondrial cytochrome b (CYTB) protein.

The early stages of speciation tend to manifest as sterility or reduced fertility in hybrids, which is thought be due to incompatibilities between genes, known as Dobzhansky-Muller incompatibilities, arising from genetic divergence between populations. Genetically characterized examples of Dobzhansky-Muller incompatibilities in animals are sparse; examples of recently diverged species, which are well-characterized and retain some behavioural capacity for interbreeding, are rare, and even where incompatible populations within a species are found, identification of the responsible genes is difficult unless the species involved are relatively amenable due to the existence of a sequenced genome, genetic markers and other genetic tools.

Where reduced hybrid fitness has been observed, asymmetry in this effect between reciprocal parental crosses is common and suggests an effect of genetic elements inherited uniparentally (example, $\mathrm{X}$ chromosome, mitochondria) (Turelli and Moyle, 2007). In plants, cytoplasmic male sterility in hybrids is commonly observed and the genetics is described in a number of cases; current mechanistic models include defective or reduced ATP production and defective cell death signalling (reviewed in Chase, 2007). But cytoplasmic male sterility in metazoa associated with naturally occurring mitochondrial variants has not been documented. This may be because it is rare; a mitonuclear interaction that produced defective sperm might be expected also to have negative effects on viability, possibly to the extent that it is incompatible with life. Or it may simply be difficult to generate in animals by crossing, and the contribution of any other uniparentally inherited genetic elements (example, sex chromosomes) must be controlled for.

Clancy (2008) generated Drosophila melanogaster strains bearing mitochondria from diverse geographic locations, but on a single isogenic nuclear background, achieved by a crossing scheme of forced chromosome replacement, using balancer chromosomes and a donor strain isogenic for chromosomes X, 2 and 3. Following the final cross, females bearing mitochondria originating from a strain from Brownsville, TX, USA, produced eggs that failed to hatch. The females were fertile, but the males were sterile in crosses with sibs and with females of a range of wild-type strains, despite apparently normal mating. The strain remains male sterile and is maintained by crossing back each generation to the isogenic donor strain, which is also being sibmated in parallel. The original Brownsville wild-type strain was not sterile; therefore, the Brownsville cytoplasm interacts with a gene from the nucleus to cause sterility. Deleterious effects mediated by exclusively maternally-transmitted genetic elements such as mitochondria should be more common in males, or even male-specific, as selection cannot operate on mitochondria in males (Frank and Hurst, 1996), but selection for nuclear modifiers to restore fertility could explain this interaction. The nuclear gene could have been on chromosomes 2, 3 or 4, but certainly not on chromosome $1(X)$ or $Y$, because fertile strains were maintained following replacement of $X$ and $Y$.

Sequencing the protein-coding mitochondrial genes revealed that Brownsville mtDNA differed from a fully fertile CR strain, Alstonville, by a single nonsynonymous nucleotide change leading to an amino acid substitution (Ala278 $\rightarrow$ Thr) in the CYTB subunit of respiratory complex III. The threonine (polar) variant is very rare, appearing in only 19 sequences, from animals ranging from heartworm to cricket, frog, gull and howler monkey, so has probably arisen independently in most cases. The alanine variant (non-polar) found in the fully fertile Alstonville strain is highly conserved; within Chordata alone, it appears in over 7800 species comprising at least 20000 sequences. The sterile males are otherwise healthy and active, although their mean lifespan is $9.6 \%(P=0.0041)$, lower than that of the Alstonville strain, largely due to excess deaths on days 10-13 (20.2 vs $10.1 \%, P=0.0004,1$-tailed Fisher exact test).

Testes from sterile males fail to individualise spermatids due to failure of formation of individualisation complex. Clusters of spermatid nuclei scatter through testes and eventually degrade in basal region of testis (Figure 1). The mechanism is unclear. Given the extensive restructuring of mitochondria in spermatids to yield the mitochondrial derivatives and the role played by mitochondria in apoptosis, spermatid individualisation may be especially sensitive to general mitochondrial dysfunction, although the flies are otherwise relatively healthy. Alternatively, the testes phenotype could be caused by oxidative damage, to which spermatozoa are particularly susceptible; they contain a large amount of mitochondria, potentially generating large amounts of superoxide, their plasma membranes are unusually rich in polyunsaturated fatty acids and so more prone to oxidation, and they lack endogenous catalase and glutathione (summarized in Kao et al., 1995). This region of CYTB catalyses ubiquinol oxidation, and the semiquinone intermediate produced at this 

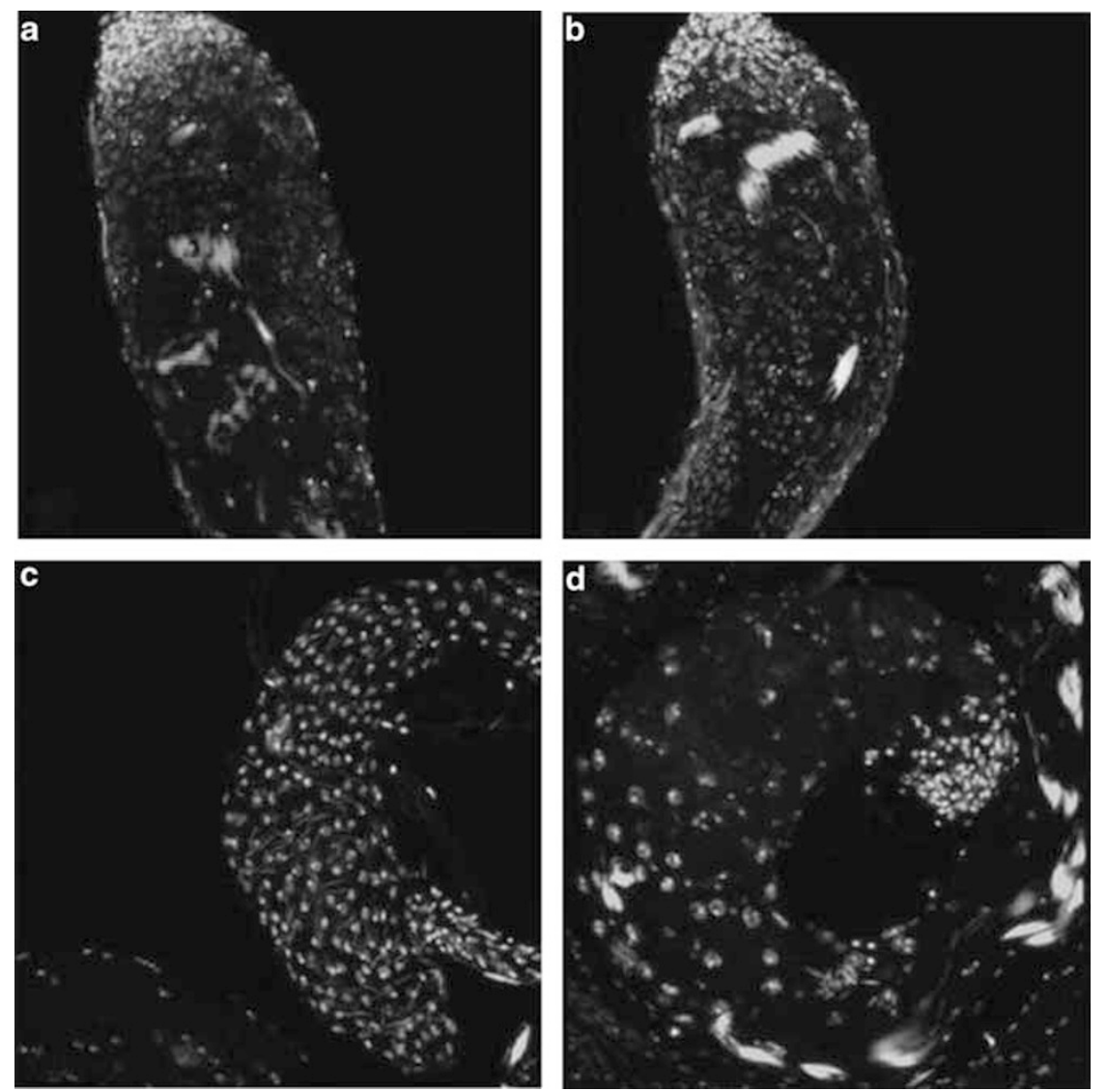

Figure 1 Absent individualisation complexes and absent mature sperm in testis from males bearing Brownsville mitochondria. Confocal photomicrographs at $\times 40$ of testis from fertile w1118 isogenic males $(\mathbf{a}, \mathbf{c})$ and sterile isogenic males bearing Brownsville mitochondria $(\mathbf{b}, \mathbf{d})$. Apical region of testis (a: control, b: sterile) stained with rhodamine-conjugated phalloidin to detect F-actin and DAPI (Invitrogen, Mulgrave, Australia) to detect DNA. Panel a (control) shows normal presence of individualisation complexes (red) and absence of clusters of spermatid nuclei (blue). Panels $\mathbf{c}$ and $\mathbf{d}$ imaged for DAPI only. Seminal vesicle (c: control) shows presence of individualised sperm nuclei (needle shaped). Base of testis (d: sterile) shows absence of individualised sperm nuclei. These were also absent from seminal vesicles. Testes were dissected and fixed as per Bunt and Hime (2004) before staining for $2 \mathrm{~h}$ with $1 \mu \mathrm{g} \mathrm{ml}{ }^{-1}$ rhodamine phalloidin (Sigma, Sydney, Australia) and mounting in ProLong Gold with DAPI (Invitrogen).

site explains the observed generation of the oxyradical superoxide; however, it is unclear how an interaction between CYTB and a nuclear gene product may result in excess oxidative effects during sperm maturation. Neither CYTB nor any naturally occurring mitochondrially-derived protein has been associated with spermatogenesis to date, and the phylogenetic distribution and conservation of $c y t b$ suggest that this functional genetic interaction may exist broadly, including mammals.

$\mathrm{Xu}$ et al. (2008) demonstrated the potential capacity of specific mitochondrial genes to affect male fertility. Using targeted germline mitochondrial expression of a restriction enzyme that cuts at a single place within the mitochondrial genome, they 'selected' for survivors carrying mtDNA point mutations, which removed the restriction site in cytochrome oxidase $I$. One of these haplotypes-conferred male sterility, seemingly without other major deleterious phenotypes. However, the amino acid sequence of this variant appears in only one sequence out of all extant (at least 20000, possibly indicating a sequencing error), so it could not be described as a naturally occurring variant in this case. Extensive work by the Burton lab (example, Ellison and Burton, 2008) has demonstrated that $F_{3}$ hybrid breakdown between allopatric populations of the marine copepod Tigriopus californicus, including reduced cytochrome c oxidase activity strongly associated with specific amino acid substitutions in the mitochondrial cytochrome c oxidase, although the sterility seemed to affect both males and females (Willett, 2008). Also, substantial maternal effect heritability for sperm mobility in fowl, where males are the homogametic sex, implicates mitochondrial genes (Froman et al., 2002), and a cytoplasmic genetic effect on sperm viability and length was demonstrated in seed beetles (Dowling et al., 2007). In humans the common mitochondrial somatic mutation, a $4977 \mathrm{bp}$ deletion, is quantitatively correlated with diminished fertility and motility of sperm (Kao et al., 1995), as is an inherited condition involving diminished activity at complexes I and IV (Folgero et al., 1993).

Cytoplasmic male sterility is likely to be seen uncommonly within a species in the lab unless forced chromosome replacement is used, or unless hybrid sterility is specifically sought. Our results, together with those discussed here, suggest that reduced male fertility due to mitonuclear incompatibilities may be much more common than previously thought, and asymmetric male subfertility in hybrids between animal populations 
may be more common than the few examples described to date.

\section{Conflict of interest}

The authors declare no conflict of interest.

\section{Acknowledgements}

This work was supported by Grant no. DP0773672 from the Australian Research Council and by the Centre for Environmental Stress and Adaptation Research. Thanks are due to Calida Neal for technical help, and to Damian Dowling and Michael Turelli for useful comments.

DJ Clancy ${ }^{1}$, GR Hime ${ }^{2}$ and AD Shirras ${ }^{1}$ ${ }^{1}$ Division of Biomedical and Life Sciences, School of Health and Medicine, Lancaster University, Lancaster, UK and ${ }^{2}$ Department of Anatomy and Cell Biology, University of Melbourne, Melbourne, Victoria, Australia E-mail: d.clancy@lancaster.ac.uk

\section{References}

Bunt SM, Hime GR (2004). Ectopic activation of Dpp signalling in the male Drosophila germline inhibits germ cell differentiation. Genesis 39: 84-93.

Chase CD (2007). Cytoplasmic male sterility: a window to the world of plant mitochondrial-nuclear interactions. Trends Genet 23: 81-90.
Clancy DJ (2008). Variation in mitochondrial genotype has substantial lifespan effects which may be modulated by nuclear background. Aging Cell 7: 795-804.

Dowling DK, Nowostawski AL, Arnqvist G (2007). Effects of cytoplasmic genes on sperm viability and sperm morphology in a seed beetle: implications for sperm competition theory? J Evol Biol 20: 358-368.

Ellison CK, Burton RS (2008). Interpopulation hybrid breakdown maps to the mitochondrial genome. Evolution 62 : 631-638.

Folgero T, Bertheussen K, Lindal S, Torbergsen T, Oian P (1993). Mitochondrial disease and reduced sperm motility. Hum Reprod 8: 1863-1868.

Frank SA, Hurst LD (1996). Mitochondria and male disease. Nature 383: 224.

Froman DP, Pizzari T, Feltmann AJ, Castillo-Juarez H, Birkhead TR (2002). Sperm mobility: mechanisms of fertilizing efficiency, genetic variation and phenotypic relationship with male status in the domestic fowl, Gallus gallus domesticus. Proc Biol Sci 269: 607-612.

Kao SH, Chao HT, Wei YH (1995). Mitochondrial deoxyribonucleic-acid 4977-bp deletion is associated with diminished fertility and motility of human sperm. Biol Reprod 52: 729-736.

Turelli M, Moyle LC (2007). Asymmetric postmating isolation: Darwin's corollary to Haldane's rule. Genetics 176: 1059-1088.

Willett CS (2008). No evidence for faster male hybrid sterility in population crosses of an intertidal copepod (Tigriopus californicus). Genetica 133: 129-136.

Xu H, DeLuca SZ, O'Farrell PH (2008). Manipulating the metazoan mitochondrial genome with targeted restriction enzymes. Science 321: 575-577. 